\title{
Excitation of Guided Acoustic Waves Using Ignition Sparks
}

\author{
Sandro Krempel, Alexander Backer, Florian Dötzer, Klaus Stefan Drese \\ Coburg University of Applied Sciences and Arts, Institute of Sensor and Actuator Technology (ISAT), \\ Am Hofbräuhaus 1b, 96450 Coburg, Germany \\ sandro.krempel@hs-coburg.de
}

\begin{abstract}
Summary:
Many sensor principles are based on the excitation and detection of guided acoustic waves (GAW). These are usually generated by mechanical coupling of piezoceramic transducers with the help of thin coupling layers. With electromechanical transducers (EMAT) or pulsed lasers, GAW can also be excited without a coupling layer or direct coupling. A further, very cost-effective possibility to excite Lamb waves on thin sheets without direct contact is shown here in the experimental setup by means of electric sparks.
\end{abstract}

Keywords: contactless excitation, guided acoustic waves (GAW), spark plug, electric spark, thermoacoustic wave excitation

\section{Introduction}

Pulsed GAW, excited by piezoceramic transducers, are frequently used in the field of material or damage analysis, but also for layer detection [1]. If individual samples are to be measured without contact, the expensive principle of thermoacoustic excitation of GAW by means of pulsed laser radiation is frequently used [2]. Korolev, Krasilnikov and Krylov have already shown that Rayleigh waves can also be excited in the material by spark discharge in air over thicker metal samples. The main effect seems to be the pressure wave generated above the material [3]. Focused pressure waves generated by sparks are also directed onto material surfaces in other places, generating bulk and Rayleigh waves $[4,5]$.

The approach presented here focuses on the experimental setup of a device using a very low-cost, commercial ignition coil and spark plug. The spark is generated directly between the spark plug and a thin metal sheet whereby Lamb waves are excited on the sheet. The excitation and propagation of the waves is detected by a laser vibrometer.

\section{Methodology}

The test setup is shown in Fig. 1. A selfdeveloped electronic switchbox (3) is supplied with $16.4 \mathrm{~V}$ voltage via a power supply EA-PS 2016-100 (1) and delivers the necessary current for charging the non-suppressed ignition coil 12V-QM50QT-6(A) (4). An Agilent 33500B function generator (2) generates a voltage pulse with a pulse width of $80 \mu \mathrm{s}$ as a control signal, which is sent to the switchbox at a re- covery rate of $20 \mathrm{~Hz}$. At the same time it also sends the trigger pulse to the laser vibrometer Polytec PSV400-M (7) to start the measurement. A suppressed iridium spark plug A7TCQM50QT-6(A) with the counter electrode removed (5) is used for spark excitation.

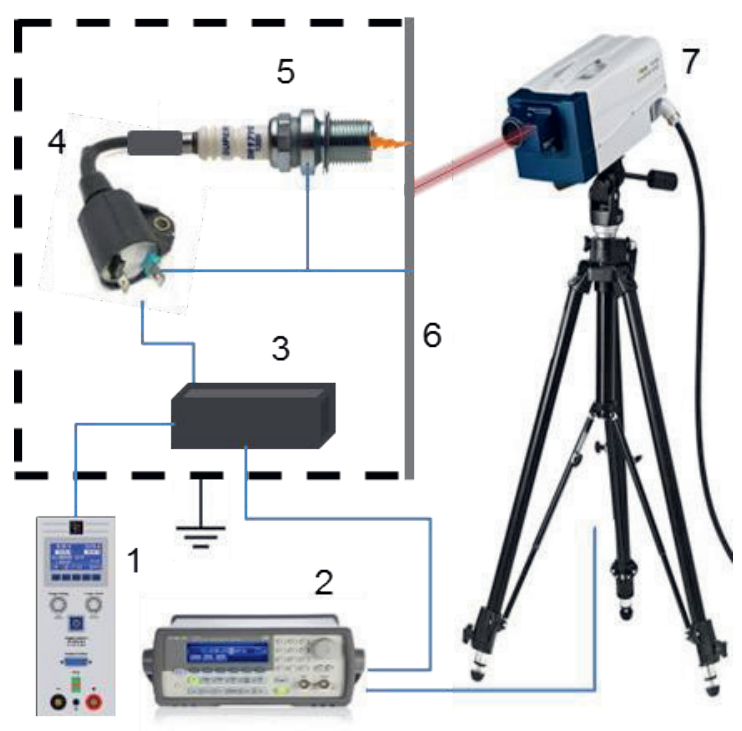

Fig.1. Illustration of the measurement setup

As the spark discharge of the spark plug causes large EM interference in the surrounding area, the switchbox as well as the coil and spark plug were installed in a closed stainless steel box. The test sample (6) is a $0.5 \mathrm{~mm}$ thick stainless steel sheet which also serves as a cover of the steel box and as a counter electrode of the spark plug. The distance of the spark plug electrode to the test sample is $0.7 \mathrm{~mm}$. To detect the generated wave, the 
outer side of the test sample is scanned with the laser vibrometer.

\section{Results and Discussion}

The vibrometer signal detected by the VD-09 velocity decoder at the point of excitation is shown in Fig. 2. After interruption of the coil current at $80 \mu \mathrm{s}$ there is a short time delay until the spark is formed, then the oscillation of the plate surface can be detected.

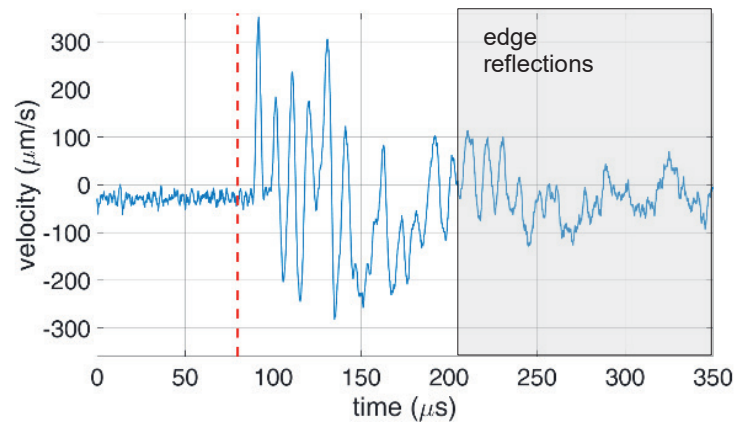

Fig.2. Time signal of the laser vibrometer measurement

From approx. 200 us onwards, interactions with edge reflections occur in the time signal. The Lamb waves excited by the point impulse show a radially symmetrical propagation (Fig. 3).

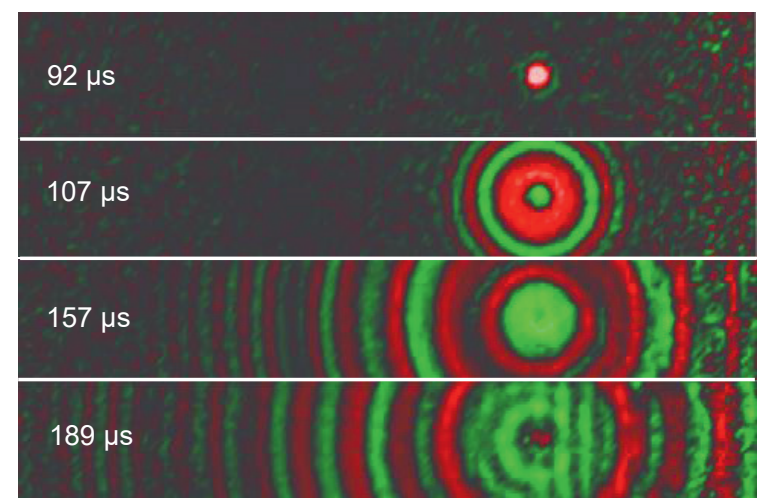

Fig.3. Surface scanning by laser vibrometer at different times.

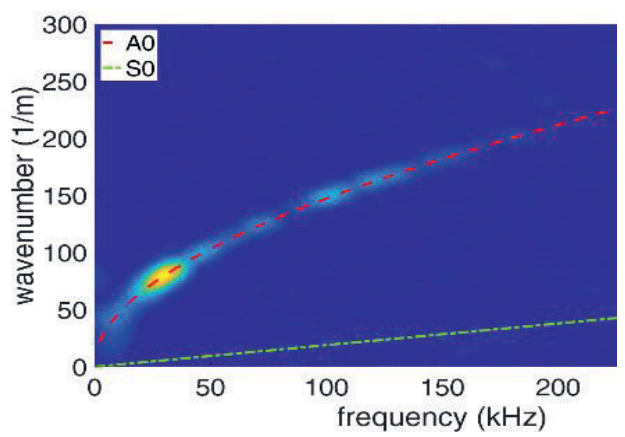

Fig.4. 2D FFT along the time and horizontal spatial axis (at the vertical center)

Using a 2D FFT of the time and horizontal position (vertical: centered) dependent from the same measurement series as used for Fig. 3, the waves excited in the measurement can be compared with the dispersion behavior of the Lamb modes calculated for a $0.5 \mathrm{~mm}$ thick stainless steel plate (Fig. 4). It can be seen that the one-sided impulse excites almost exclusively the antisymmetric A0 mode. In addition to the dominant frequency of approx. $30 \mathrm{kHz}$ frequency components up to approx. $200 \mathrm{kHz}$ are also visible.

\section{Conclusion and outlook}

The experimental investigations have shown that even with a very simple and inexpensive ignition coil - spark plug design $(<100 €)$ it is possible to excite guided acoustic waves in a metal plate without contact and broadband, but above all at very low frequencies. This would, for example, open up new possibilities in the field of sensor technology for structural health monitoring. In further investigations, the following points are to be clarified more precisely: local position accuracy and repeatability of the spark impact, potential material removal on the sample, electrode geometry and position relative to the sample, extension of the frequency range, investigation of the contribution of various excitation mechanisms to the formation of GAWs (thermal expansion, pressure waves).

\section{Acknowledgments}

The authors thank the various supporters of the project at ISAT, especially Frank Ritter, Tobias Köstner, Andrej Schumacher, Sabrina Tietze and the European Fund of Regional Development (EFRE) within the project "InnoTerm".

\section{References}

[1] G. Lindner, M. Schmitt, K. Schmidt, H. Faustmann, S. Krempel, J. Schubert, Detection of coatings and measurement of coating thickness on technical substrates using surface acoustic waves in a waveguide configuration, Proc. Sensor 2009 (1), 35-40 (2009); doi: 10.5162/sensor09/v1/a1.3

[2] F. Singer, Laser-Acoustic Characterization of Coatings, AMA Conferences 2015 - SENSOR 2015 and IRS2 2015, 119-123; doi: 10.5162/sensor2015/A5

[3] S. V. Korolev, V. A. Krasilnikov, V. V. Krylov, Mechanism of sound generation in a solid by a spark discharge near the surface, Sov. Phys. Acoust. 33 (4), 774-776, (1987)

[4] X. Dai, J. Zhu, M. Haberman, A focused electric spark source for non-contact stress wave excitation in solids, JASA Express Letters, 2013; doi: $10.1121 / 1.4826913$

[5] S. V. Korolev, V. V. Krylov, Efficient excitation of Rayleigh waves by a strong shock wave initiated by a spark in air, Sov. Tech. Phys. Lett. 14 (11), 1945-1949, (1988) 